\title{
Optimizing Rehabilitation and Return to Sport in Athletes With Anterior Knee Pain Using a Biomechanical Perspective
}

\author{
Brian J. Theisen, P.T., D.P.T., S.C.S., A.T.C., Peter D. Larson, P.T., D.P.T., O.C.S., and \\ Caitlin C. Chambers, M.D.
}

\begin{abstract}
Anterior knee pain represents one of the most common athletic knee conditions and arguably also one of the most complex. The patellofemoral joint is at the center of several forces, and alterations in any of these force vectors due to muscular imbalance, soft-tissue tightness or laxity, and altered functional movement patterns can all combine to create a painful anterior knee. While typically anterior knee pain is not a surgical entity, the orthopaedic surgeon with an understanding of these biomechanical intricacies is best positioned to provide comprehensive evidence-based care for the patient with anterior knee pain. Level of Evidence: V, expert opinion.
\end{abstract}

A nterior knee pain (AKP) is one of the most common sport-related knee conditions. ${ }^{1,2}$ While often referred to as patellofemoral pain, the true pathophysiologic origin of AKP is not always rooted in abnormalities of the patella or femoral trochlea but can be due to strain experienced by any of the surrounding innervated structures, such as the infrapatellar fat pad, medial and lateral retinaculum, or synovium. ${ }^{3-5}$ The dominant theory explaining the genesis of AKP focuses on the issue of excessive joint loading and elevated patellofemoral joint stress (PFJS) resulting in increased strain to these innervated structures in the anterior knee. ${ }^{4-7}$ In addition, aberrations in both peripheral and central pain-processing mechanisms can be seen in patients with AKP, with generalized hyperalgesia and pain catastrophization seen in some with AKP.,8-11 While classically described as a self-limited condition,

From the TRIA Orthopedic Center, Woodbury (B.J.T., P.D.L., C.C.C.); and Department of Orthopedic Surgery, University of Minnesota, Minneapolis (C.C.C.), Minnesota, U.S.A.

The authors report that they have no conflicts of interest in the authorship and publication of this article. Full ICMJE author disclosure forms are available for this article online, as supplementary material.

Received August 26, 2021; accepted October 18, 2021.

Address correspondence to Caitlin C. Chambers, M.D., Department of Orthopedic Surgery, University of Minnesota, 2450 Riverside Ave., South Minneapolis, MN 55454.E-mail: cchamber@umn.edu

(C) 2021 THE AUTHORS. Published by Elsevier Inc. on behalf of the Arthroscopy Association of North America. This is an open access article under the CC BY-NC-ND license (http://creativecommons.org/licenses/by-nc-nd/4.0/). 2666-061X/211253

https://doi.org/10.1016/j.asmr.2021.10.028
AKP can become a devastatingly persistent and recurrent chronic problem. ${ }^{12-15}$ Relatively few patients with AKP ultimately require surgery, with multimodal nonoperative treatment including physical therapy remaining the mainstay of treatment for a vast majority. As such, familiarity of practitioners with appropriate evidence-based rehabilitation plans is paramount to successful treatment of patients experiencing AKP. In this manuscript, we aim to summarize the key variables worthy of consideration in the treatment of patients with a primary complaint of AKP (Fig 1).

\section{Biomechanical Considerations in AKP}

Biomechanical risk factors for AKP include those which are either anatomic, due to structural malalignment, or functional, due to altered lower extremity kinematics. Anatomic risk factors including trochlear dysplasia, patella alta, genu valgum, a lateralized tibial tubercle, increased femoral anteversion or external tibial torsion, and an overpronated foot posture can all play a contributing role in AKP but will not be the focus of this manuscript. Functional risk factors for AKP arise due to abnormal movement patterns and require the attention of a skilled physical therapist to restore more balanced joint loading in an effort to improve pain and function. These functional risk factors include altered lower-extremity joint kinematics, decreased muscle strength, increased musculotendinous tightness, and altered neuromuscular recruitment. ${ }^{5,16}$ In particular, improper hip and knee frontal plane dynamic alignment have been reported as contributors to AKP. ${ }^{17-19}$ 


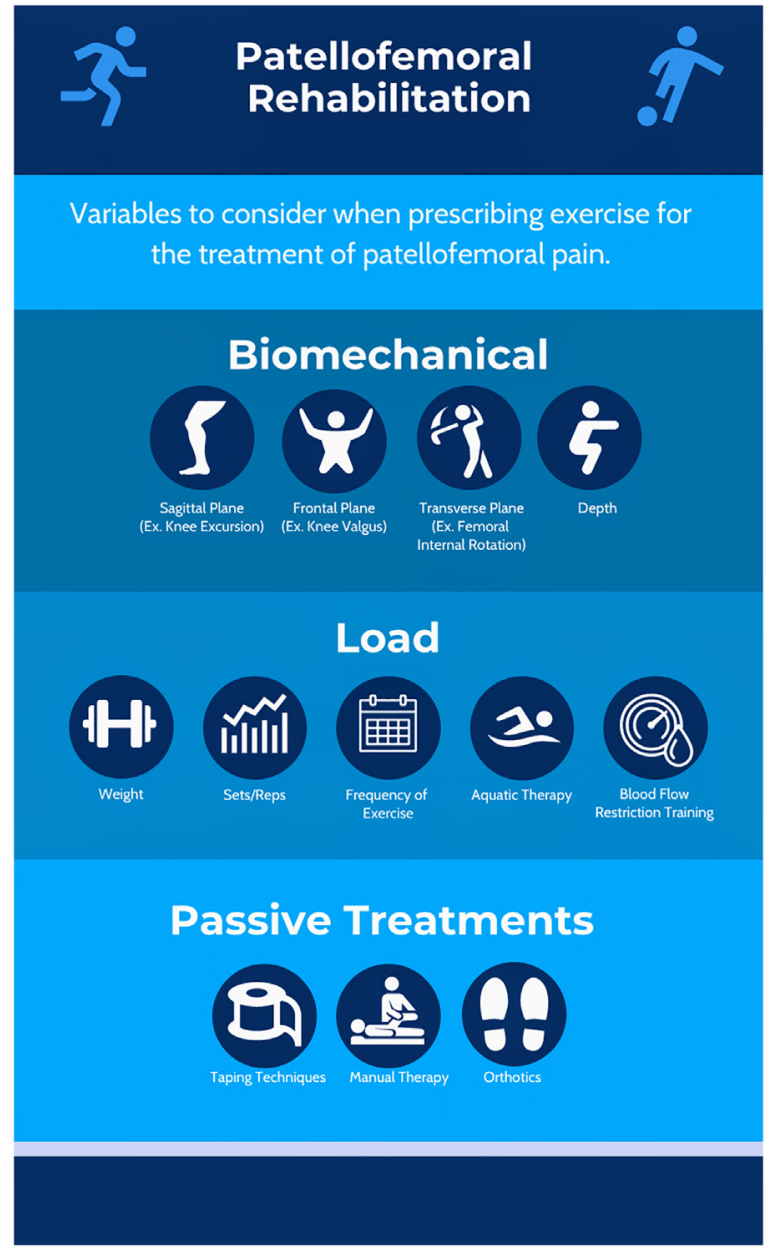

Fig 1. Key variables worthy of consideration in the treatment of patients with a primary complaint of anterior knee pain.

Dynamic valgus is a pathologic movement pattern that presents as a valgus lower-extremity positioning seen during weighted flexion activities, in particular those isolating a limb individually such as stair climbing, running, and jump landing. This movement pattern results in disproportionate loading of the lateral patellofemoral joint, reducing patellofemoral contact area thus increasing focal joint stress laterally, while also creating tightness of lateral soft-tissue structures if present long-term. ${ }^{4}$ The kinematic factors found to impart the greatest influence on dynamic valgus include increased hip adduction and increased hip internal rotation affecting frontal and transverse plane knee motion, respectively. ${ }^{4}$ Even $5^{\circ}$ of excessive femoral internal rotation has been shown to increase patella cartilage stress during a squatting task, ${ }^{20}$ and increased hip internal rotation ${ }^{21}$ and dynamic knee valgus during single-leg squat and jump landing have been correlated with AKP, particularly in female patients. ${ }^{17,22,23}$ Holden et al. $^{22}$ found that knee valgus displacement measured at $\geq 10.6^{\circ}$ during a jump landing task predicted AKP in adolescent female patients with a sensitivity of 0.75 and specificity of 0.85 and appeared to represent a manifestation of altered motions at the hip. In addition, individuals with AKP more often exhibit an ipsilateral trunk lean during singlelegged squat and jump landing tasks, which shifts the center of mass toward the stance limb and increases potential for dynamic valgus. ${ }^{21,24}$ Suffice it to say that proximal hip and trunk strength play a large role in dynamic valgus and AKP and should thus be objectively assessed and targeted by clinicians caring for patients with AKP. Indeed, current evidence supports a combination of hip- and knee-strengthening exercises rather than knee exercises alone in order to improve pain and function most effectively in patients with AKP. ${ }^{25-27}$

Decreased flexibility of the quadriceps, hamstrings, iliotibial band, gastrocnemius, and soleus have been implicated as risk factors for AKP due to a theoretical increase in patellar compression, but findings are inconsistent among high-quality studies, so no direct relationship is currently regarded as consensus., 5,28 Excessive tightness of any lower-extremity muscular group identified by a clinician should certainly be included as a target in the overall treatment plan.

Overall, a multimodal approach is recommended in treating patients with AKP. ${ }^{25,29}$ This should include patient education, physical therapy targeting hip and knee musculature, correction of pathomechanics, and the addition of other modalities based upon individual needs, including patellar taping, joint mobilizations, or neuromuscular electrical stimulation. ${ }^{25,29}$

\section{PRJFS in Strengthening}

The most effective strategy in addressing AKP through rehabilitation centers around strengthening of the hip and thigh musculature, as both have been found to significantly reduce pain and improve function in the short-, medium-, and long-term. ${ }^{30}$ However, the clinician must also be mindful that the prescribed exercises do not excessively increase AKP, as this can be a deterrent to therapy compliance. ${ }^{31}$ Clinicians should be attentive to how specific exercises affect PFJS, as it is hypothesized that either excessive peak PFJS or cumulative PFJS is a crucial component in the cascade leading to AKP. Understanding the principle of PFJS is paramount to preventing its excess, with stress defined as force per area; either an increase in force or decrease in contact area yields increased PFJS. Contact area between the patella and trochlea can greatly vary depending on several modifiable and nonmodifiable factors. ${ }^{4}$ Understanding these changes based on the activity and lower-extremity biomechanics can help clinicians prescribe appropriately challenging exercises that fatigue the targeted muscle while minimizing PFJS and resultant pain. 
Table 1. Ways to Decrease PFJS During CKC Exercises

\begin{tabular}{|c|c|c|c|}
\hline & Hip/Trunk & Knee & Ankle/Foot \\
\hline$\overline{\text { Sagittal }}$ & Forward trunk lean & Minimize anterior translation of knee & Weight through heel \\
\hline Frontal & Hip neutral (no lateral trunk lean) & $\begin{array}{l}\text { Knee in line with middle of foot (avoid } \\
\text { dynamic valgus) }\end{array}$ & Neutral arch \\
\hline Transverse & Hip neutral (no trunk rotation) & $\begin{array}{l}\text { Knee in line with middle of foot (avoid } \\
\text { femoral internal rotation) }\end{array}$ & $\begin{array}{l}\text { ER of foot to compensate for excessive } \\
\text { femoral anteversion/internal tibial } \\
\text { torsion. } \\
\text { IR of foot to compensate for excessive } \\
\text { femoral retroversion/external tibial } \\
\text { torsion }\end{array}$ \\
\hline
\end{tabular}

CKC, closed kinetic chain; ER, external rotation; IR, Internal rotation; PFJS, patellofemoral joint stress.

\section{Open Kinetic Chain (OKC) Versus Closed Kinetic Chain (CKC)}

Powers et al. $^{32}$ found that when strengthening the quadriceps in the OKC using an ankle weight or knee extension machine with a variable moment arm, the patellofemoral joint experiences relatively lower PFJS from $90^{\circ}$ to $45^{\circ}$ of knee flexion, and higher PFJS from $45^{\circ}$ to $0^{\circ}$ of knee extension. This is due to both the increasing moment arm and decreasing patellofemoral joint contact area as the knee extends. When using a knee extension machine with a fixed moment arm, PFJS is consistently greater throughout the entire $90^{\circ}$ to $0^{\circ}$ range of motion. ${ }^{32}$ In the closed kinetic chain $(\mathrm{CKC})$, PFJS is relatively lower from $0^{\circ}$ to $45^{\circ}$ of knee flexion, increases from $45^{\circ}$ to $90^{\circ}$, then levels off from $90^{\circ}$ and onward. ${ }^{32}$ These factors must be considered when constructing a rehabilitative plan for patients with AKP who may be particularly sensitive to an increase in PFJS with certain exercises. In such a scenario, it is our general recommendation to use short-arc CKC $\left(0^{\circ}-45^{\circ}\right)$ and OKC $\left(90^{\circ}-45^{\circ}\right)$ exercises in the early range of knee flexion for patients actively complaining of AKP in which load reduction is desired.

\section{Lower-Extremity Mechanics in the Cardinal Planes}

Clinicians should be aware of how differing lowerextremity biomechanics in each of the cardinal planes will alter PFJS, as it is important to be able to identify and correct alterations in form that may be contributing to a patient's AKP. Table 1 summarizes strategies that clinicians can use to reduce PFJS by modifying hip, knee, and ankle or foot mechanics. It is important to remember that while minimizing PFJS should be a focal point of rehabilitation when AKP levels are high, as pain decreases, clinicians may want to consciously begin increasing PFJS to help improve the patient's load tolerance.

In the sagittal plane, forward trunk lean, anterior knee excursion, and ankle dorsiflexion mobility can all impact PFJS. ${ }^{28,33-35}$ PFJS can be reduced by increasing forward trunk lean during CKC knee-strengthening exercises. Escamilla et al. $^{34}$ found that compared with wall squats, ascending from a single-leg squat creates less PFJS from $90^{\circ}$ to $60^{\circ}$. They surmised that this is due to the lack of hip motion during wall squats, transitioning much of the force to the knee. In addition, lunging with a shortened stride, which results in anterior knee excursion beyond the toes, increases PFJS significantly more than a long stride lunge which keeps the tibia more vertical at the bottom of the motion. ${ }^{36}$ The clinician should therefore assess patients performing squats and single limb exercises from a lateral view to appreciate whether these tendencies exist in a symptomatic knee. If present, clinicians should provide appropriate feedback and assess whether symptoms reduce with the altered movement pattern. Clinicians

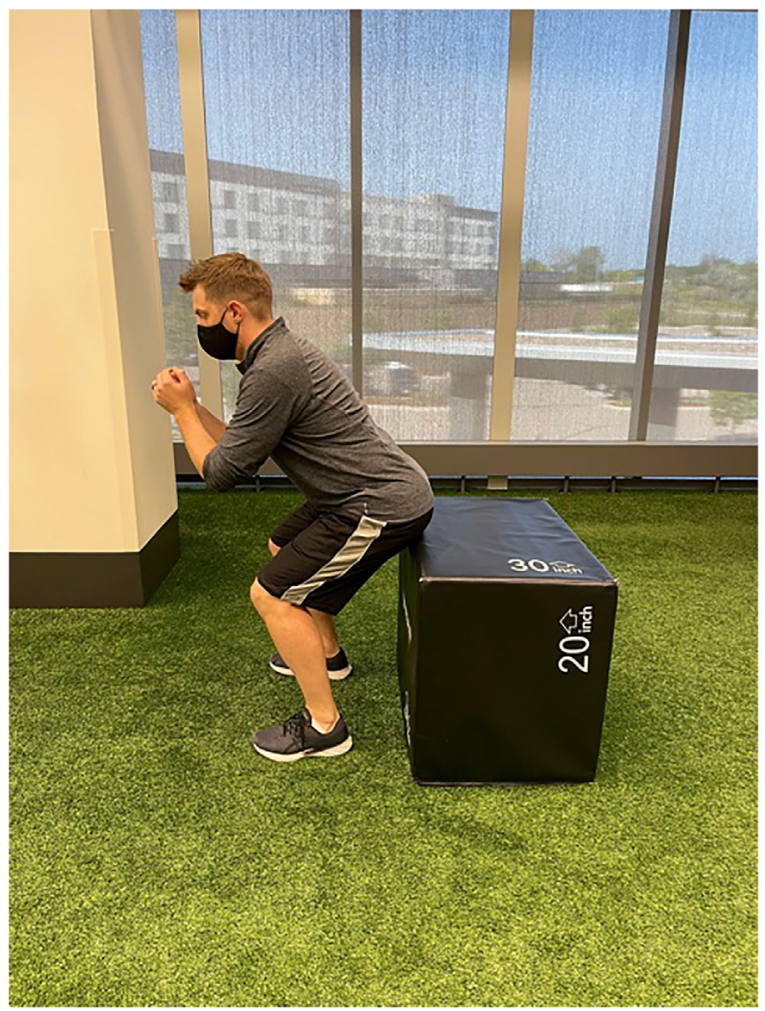

Fig 2. External cue to increase trunk flexion. 


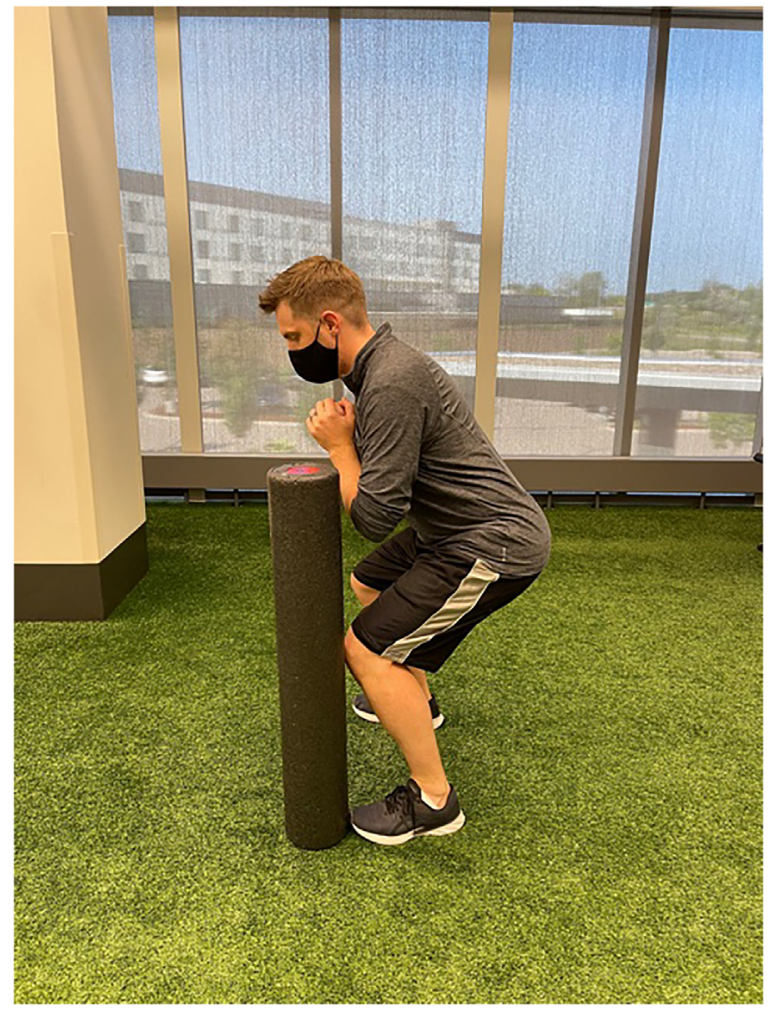

Fig 3. External cue to decrease excessive anterior knee excursion.

should consider using external feedback, such as a mirror, video analysis, or a physical object to help provide feedback, versus internal feedback (e.g., "flex your trunk more"), as this has been shown to enhance motor performance. ${ }^{37}$ A simple external cue to increase trunk flexion would be to place a plyometric box behind the patient for them to sit their hips back toward (Fig 2). To limit excessive anterior knee excursion, clinicians can use a foam roller or dowel to physically block the knee from going beyond the toes (Fig 3).

In the transverse plane, tibiofemoral rotation angle has a significant effect on PFJ contact area and thus PFJS in those with AKP. ${ }^{21,38}$ During closed-chain exercises and weight-bearing activities, the tibia is relatively fixed while the femur is free to rotate. Patients with AKP commonly demonstrate increased femoral internal rotation during single-leg squat exercises, with studies showing nearly twice the amount of rotation as seen in controls without AKP. ${ }^{39,40}$ Accordingly, teaching patients with AKP control in minimizing femoral internal rotation during jump-landing and closed chain squatting exercises can prove a particularly useful biomechanical adjustment with the potential to impart significant reduction in PFJS and resultant anterior knee pain.

In the frontal plane, ipsilateral trunk lean as well as varus or valgus positioning of the knee alter PFJS. ${ }^{21,23}$ In particular, increased knee adduction, resulting in

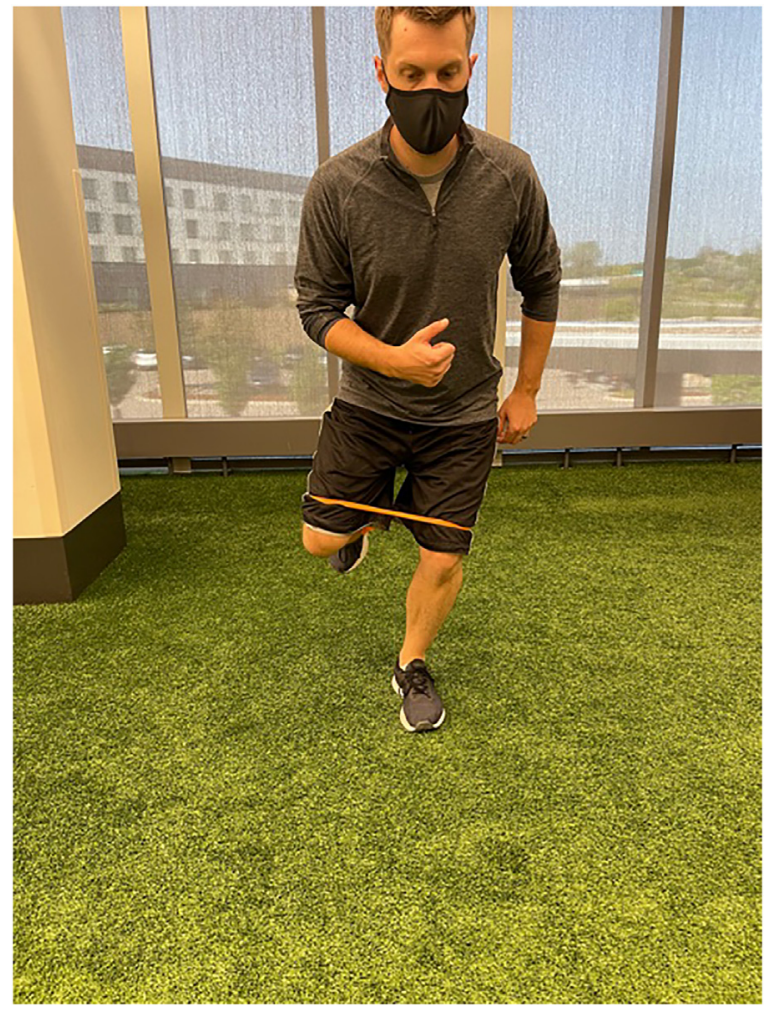

Fig 4. Exercise band for those with excessive genu valgum and/or femoral internal rotation.

genu valgum, loads the lateral PFJ, while the excessive knee abduction in genu varum loads the medial PFJ. ${ }^{41,42}$ Ipsilateral lateral trunk lean and knee adduction can both be related to weakness of hip musculature, particularly the gluteus medius, thus warranting guided proximal muscular strengthening if this movement pattern is seen. To properly assess both transverse and frontal plane kinematics, clinicians should observe squats and single-limb exercises from an anterior view. Improved knee positioning in the frontal and transverse plane can be achieved by using an exercise band looped around both thighs for those with excessive genu valgum and/or femoral internal rotation (Fig 4). To aid in trunk positioning, the patient can hold a dowel in the contralateral arm and/or use mirror feedback to maintain a more erect posture and avoid lateral trunk lean (Fig 5).

\section{Recommendations Regarding Strengthening and Periodization}

The goal of rehabilitation in AKP is to strengthen the quadriceps and hips without excessively increasing AKP. The first step for the clinician is to objectively determine which muscle groups would primarily benefit from strengthening. It is recommended that the clinician assess strength using isokinetic machine or handheld dynamometer, testing the quadriceps, 


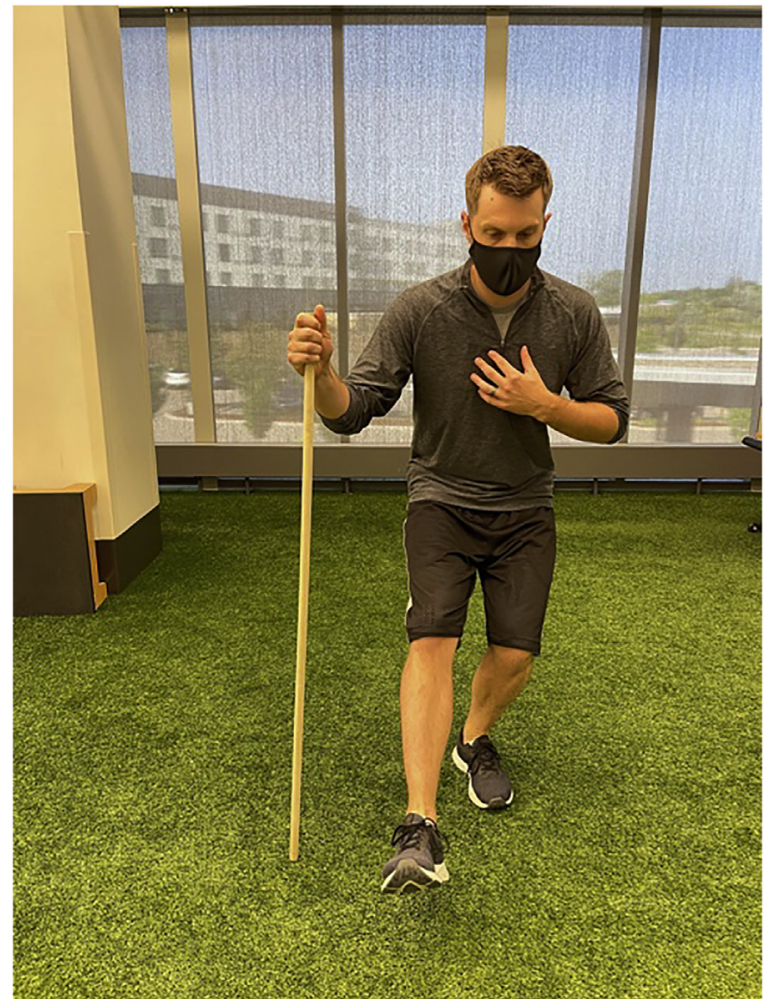

Fig 5. Dowel on contralateral side to decrease excessive ipsilateral and forward trunk lean.

hamstrings, and hip muscles bilaterally, looking for side-to-side differences. This will help guide early prescription of exercises, since preferential treatment should be given to the relatively weakest muscles. In patients who have relative quadriceps weakness, exercise prescription should start in the following manner: OKC knee extension can be initiated with a variable moment arm from $90^{\circ}$ to $45^{\circ}$ and in the CKC from $0^{\circ}$ to $45^{\circ}$, or in positions with relatively minimal PFJS, such as straight leg raises. For those with relative hip weakness, rehabilitation should focus specifically on hip strengthening in the earlier stages. As symptoms and strength improve, the clinician can then progress patients into further ranges of motion that increase PFJS, guided by patient pain levels both during and after completion of the exercises.

Consideration must also be given to the dose of strengthening exercises in terms of repetitions, sets, and frequency. Initially, it is recommended the clinician start with greater repetitions; this is done for 3 reasons. First, some patients have intolerance to high absolute PFJS, and therefore by starting with lighter loads and greater repetitions, the absolute PFJS can be mitigated while still inducing muscle fatigue and subsequent strength gains. Second, Dinyer et al. ${ }^{43}$ have shown that perhaps fatigue itself, and not fatigue in a certain repetition window, is the largest driver of strength gains in untrained individuals. Finally, it is common in

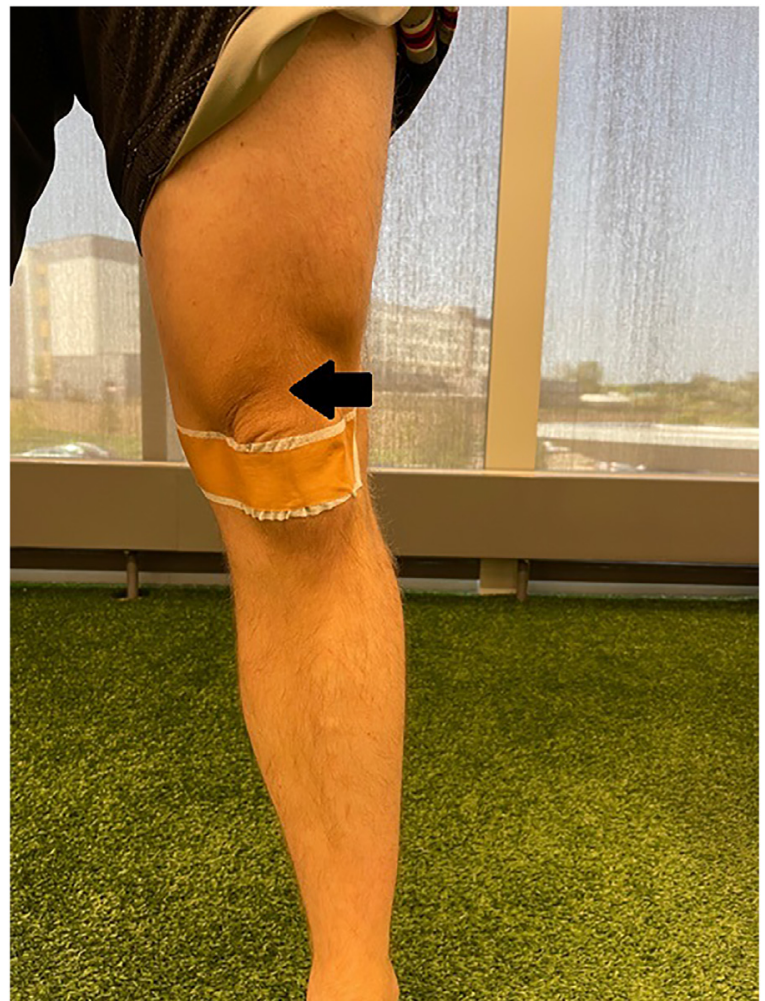

Fig 6. Medial McConnell taping, with tape applied pulling from lateral to medial, to reduce symptoms attributed to lateral patellofemoral overload by more evenly distributing forces across the patellofemoral joint.

strength training to start with lighter loads and greater repetitions, as it is safer for novices who are learning correct mechanics to minimize risk of additional injury. At the onset of rehabilitation, patients should be encouraged to perform exercises that fatigue at $10+$ repetitions. To ensure proper difficulty, the clinician should consider monitoring the patients' reported repetitions in reserve and/or rate of perceived exertion after each set, then alter the exercise appropriately. The number of sets prescribed should be determined based on the overload principle of strengthening, as well as the patient's current activity level. The overload principle is the fundamental theory of training in which exercise at an intensity above that normally attained will induce highly specific adaptations, enabling the body to function more efficiently.

Frequency of exercise performance should also be a consideration. For exercises that put relatively little stress on the PFJ and are done at higher repetition schemes, the clinician can consider prescribing daily, or even multiple times per day. As exercise intensity increases, the clinician should consider scaling back the frequency of exercise to model more typical strengthening programs, such as 2-3 times per week. ${ }^{44}$ Finally, patients and clinicians should collaboratively agree on acceptable pain levels during and after exercise 
performance, understanding that hurt does not necessarily equal harm, and some levels of pain are acceptable. With all variables, a collaborative decision between the rehabilitation specialist and the patient should be made based on patient feedback, symptoms, and the overload principle.

\section{Modality Use in AKP Rehabilitation}

Exercise-based interventions are the backbone of evidence-based treatment for AKP, but there can be indications for use of additional modalities. Adjunct modalities such as patellofemoral taping, blood flow restriction (BFR) therapy, neuromuscular electrical stimulation, and manual therapy can serve to make exercise more comfortable, improve biomechanics and increase patient buy-in to physical therapy.

The evidence regarding efficacy of patellofemoral taping is inconclusive, however with appropriate indications, it can be helpful to clinicians in several ways. ${ }^{45-47}$ Patellofemoral taping can make exercisebased treatments more comfortable, increase patient buy-in, and serve as a diagnostic and prognostic tool when surgery is under consideration. Patellofemoral taping is most effectively used by assessing a functional task pre- and post-taping that correlates with the patient's symptoms (i.e., stair climbing); if pain is alleviated after the application of tape, then taping may be warranted in the short term to help the patient complete functional activities and exercises that may otherwise be painful. In particular, medial tilt and medial glide McConnell taping (Fig 6) can help to alleviate pain when the underlying cause is lateral patellofemoral overload. This technique applies a medial force vector on the patella, resulting in a more even distribution of joint forces over a larger contact area. ${ }^{47,48}$ Improved pain and function with use of medial tilt or medial glide McConnell taping can be seen as a positive prognostic indicator for surgeons when considering a lateral unloading procedure (lateral retinacular lengthening or release, anteromedializing tibial tubercle osteotomy) for treatment of lateral patellofemoral overload. Lack of response to medial taping techniques may implicate lesser functional improvements post-operatively, and warrant consideration of other pain sources before undertaking a lateral unloading procedure.

BFR can be a very helpful tool in the treatment of anterior knee pain. Several studies have highlighted the importance of quadriceps strengthening to reduce pain and improve function in patients with AKP; however, quadriceps strengthening exercises also can exacerbate knee symptoms if they exceed joint load tolerance capacities. ${ }^{49,50}$ This creates a significant challenge to clinicians when it comes to determining appropriate loads to increase muscle function without provoking symptoms. BFR can help with this challenge both promoting muscle hypertrophy at lower loads and producing a short-term reduction in pain. Evidence suggests that low loads (20\%-30\% lRM) with BFR can induce benefits in regards to knee pain, functional capacities, quadriceps strength, and thickness in patients with knee conditions in comparison with a low-load training program. ${ }^{51}$ BFR is also an effective treatment option for patients who are struggling to tolerate advancements in CKC activities. Compared with standard quadriceps strengthening, low load with BFR produced greater reduction in pain with daily living at 8 weeks in people with AKP. ${ }^{51}$ In addition, BFR may provide a short-term hypoalgesic effect, as Korakakis et al. ${ }^{52}$ found that lowlevel resistance training with BFR significantly reduced pain in functional activities immediately postintervention for at least 45 minutes. Starting a physical therapy session with a well-tolerated exercise and BFR can be used to mitigate symptoms with more advanced exercises immediately following.

While manual therapy alone has not been supported by research, when used in conjunction with exercise, it may be helpful for relief of AKP in the short term. ${ }^{53}$ Manual therapy such as joint mobilizations can be beneficial especially in cases where mobility deficits (i.e., quadriceps, ankle dorsiflexion) or excessive lateral pressure are contributors however should not compromise time spent on more evidence-supported exercise interventions.

Insufficient evidence exists in the support of global use of neuromuscular electrical stimulation (NMES) in patients with AKP; however, if the patient presents with poor quadriceps activation and/or strength, NMES can help to address these deficits. With a healthy quadriceps set, the patella should translate approximately $10 \mathrm{~mm}$ superiorly, but with poor quadriceps activation, deficient superior glide of the patella is seen resulting in increased joint compression. ${ }^{54}$ A 2010 systematic review on NMES on quadriceps strength in individuals after anterior cruciate ligament reconstruction found that NMES combined with exercise is more effective than exercise alone at improving quadriceps muscle strength. ${ }^{55}$

\section{Return to Sport}

For AKP, the return to sport phase of rehabilitation and the associated decision-making closely resembles that of other knee injuries and surgeries. This phase should involve functional testing, progressive sportspecific drills, and graduated practice participation. Hop testing, dynamometry or isokinetic testing, functional strength tests such as single-leg squats and singleleg bridges should be used to determine quantitative limb symmetry. Achieving limb symmetry is imperative in the AKP population; however, clinicians often do not hold this population to the same standards as postsurgical patients. When the athlete is currently 
participating in their sport, as opposed to being cleared to participate postoperatively, the decision-making regarding return to sport can be convoluted by an athlete's desire or motivation to continue participating in their sport, regardless of current symptoms or risk of future injury. Patient education regarding their strength deficits and its impact on quality of movement and associated injury risk is important. Assessing the quality of movement throughout the functional test is equally as important as the quantitative results. Video analysis is especially helpful in identifying faults and illustrating them to patients. While frontal and transverse plane faults are certainly common in this population, the importance of assessing for sagittal plane movement faults is critical as this will often reveal quadricepsavoidant movement patterns, which are particularly impactful in the deceleration activities of cutting and jumping sports. The sagittal plane view of landings on single-leg hop tests commonly expose compensatory patterns including lower knee flexion angles at landing, excessive anterior and ipsilateral trunk lean, and less ankle power absorption. ${ }^{56}$ Anterior knee pain is commonly seen bilaterally which can make limb symmetry numbers less reliable. In these cases, normative data can be helpful in clinical decision making. Normative data exist for hop testing and isokinetic testing; however, this is an area in which further research with specific populations would be helpful.

While assessing limb symmetry and biomechanical faults are important in determining readiness for to return to sport, the management of training loads and athlete capacity often play a larger role in a successful return by athletes with patellofemoral pain. ${ }^{57}$ Dye $^{58}$ proposed a tissue homeostasis model for the patellofemoral joint, suggesting that the patellofemoral joint requires loads to be applied in correspondence to the maximum capacity the body can tolerate and recover from. Training in excess of the load threshold in either intensity or frequency disrupts tissue homeostasis resulting in AKP symptoms. Patient education about load management and activity modification is therefore crucial when beginning the return to sport phase. Athletes should engage in progressive practice participation before competition. Rathleff et al. ${ }^{59}$ demonstrated efficacy of activity modifications according to symptoms and associated return to sport guidance in active adolescents with AKP. Participants were instructed to gradually increase activities only if pain levels did not exceed 2 of 10 in severity. Immediately after their 12 -week intervention, $86 \%$ of participants reported a successful treatment outcome, and success rates remained high at 6 months $(77 \%)$ and 12 months $(81 \%) .^{59}$

\section{Conclusions}

Anterior knee pain is a condition that is certain to be encountered by any sports medicine clinician and that can prove frustrating to the patient and clinician alike due to the complexities involved in addressing contributors to pain. Proper evaluation and management of the many factors at play including altered biomechanics, muscular weakness, soft-tissue tightness, and neuromuscular activation patterns is crucial in providing appropriate treatment to patients suffering from AKP. Familiarity with methods of reducing joint loads in early rehabilitation can be helpful in sustaining patient buy-in by reducing their pain throughout the therapeutic exercises necessary for long-term pain relief.

\section{References}

1. Baquie P, Brukner P. Injuries presenting to an Australian sports medicine centre: A 12-month study. Clin J Sport Med 1997;7:28-31.

2. Taunton JE, Ryan MB, Clement DB, McKenzie DC, LloydSmith DR, Zumbo BD. A retrospective case-control analysis of 2002 running injuries. Br J Sports Med 2002;36: 95-101.

3. Post WR, Dye SF. Patellofemoral pain: An enigma explained by homeostasis and common sense. Am J Orthop (Belle Mead, NJ) 2017;46:92-100.

4. Powers CM, Bolgla LA, Callaghan MJ, Collins N, Sheehan FT. Patellofemoral pain: Proximal, distal, and local factors, 2nd International Research Retreat. J Orthop Sports Phys Ther 2012;42:A1-54.

5. Powers CM, Witvrouw E, Davis IS, Crossley KM. Evidence-based framework for a pathomechanical model of patellofemoral pain: 2017 patellofemoral pain consensus statement from the 4th International Patellofemoral Pain Research Retreat, Manchester, UK: Part 3. Br J Sports Med 2017;51:1713-1723.

6. Dye SF. The pathophysiology of patellofemoral pain: A tissue homeostasis perspective. Clin Orthop Rel Res 2005;(436):100-110.

7. Farrokhi S, Keyak JH, Powers CM. Individuals with patellofemoral pain exhibit greater patellofemoral joint stress: A finite element analysis study. Osteoarthritis Cartilage 201 1;19:287-294.

8. Lack S, Neal B, De Oliveira Silva D, Barton C. How to manage patellofemoral pain-Understanding the multifactorial nature and treatment options. Phys Ther Sport 2018;32:155-166.

9. Rathleff MS, Vicenzino B, Middelkoop M, et al. Patellofemoral pain in adolescence and adulthood: Same same, but different? Sports Med 2015;45:1489-1495.

10. van der Heijden RA, Rijndertse MM, BiermaZeinstra SMA, van Middelkoop M. Lower pressure pain thresholds in patellofemoral pain patients, especially in female patients: A cross-sectional case-control study. Pain Med 2018;19:184-192.

11. Bartholomew C, Lack S, Neal B. Altered pain processing and sensitisation is evident in adults with patellofemoral pain: A systematic review including meta-analysis and meta-regression. Scand J Pain 2019.

12. Collins NJ, Bierma-Zeinstra SM, Crossley KM, van Linschoten RL, Vicenzino $B$, van Middelkoop $M$. 
Prognostic factors for patellofemoral pain: A multicentre observational analysis. Br J Sports Med 2013;47:227-233.

13. Crossley KM, van Middelkoop M, Callaghan MJ, Collins NJ, Rathleff MS, Barton CJ. 2016 Patellofemoral pain consensus statement from the 4th International Patellofemoral Pain Research Retreat, Manchester. Part 2: Recommended physical interventions (exercise, taping, bracing, foot orthoses and combined interventions). $\mathrm{Br} J$ Sports Med 2016;50:844-852.

14. Lankhorst NE, van Middelkoop M, Crossley KM, et al. Factors that predict a poor outcome 5-8 years after the diagnosis of patellofemoral pain: A multicentre observational analysis. Br J Sports Med 2016;50:881-886.

15. Rathleff MS, Rathleff CR, Olesen JL, Rasmussen S, Roos EM. Is knee pain during adolescence a self-limiting condition? Prognosis of patellofemoral pain and other types of knee pain. Am J Sports Med 2016;44:1165-1171.

16. Bolgla LA, Boling MC, Mace KL, DiStefano MJ, Fithian DC, Powers CM. National Athletic Trainers' Association Position Statement: Management of individuals with patellofemoral pain. J Athl Training 2018;53: 820-836.

17. Myer GD, Ford KR, Barber Foss KD, et al. The incidence and potential pathomechanics of patellofemoral pain in female athletes. Clin Biomech (Bristol, Avon) 2010;25: 700-707.

18. Myer GD, Ford KR, Di Stasi SL, Foss KD, Micheli LJ, Hewett TE. High knee abduction moments are common risk factors for patellofemoral pain (PFP) and anterior cruciate ligament (ACL) injury in girls: Is PFP itself a predictor for subsequent ACL injury? Br J Sports Med 2015;49:118-222.

19. Noehren B, Hamill J, Davis I. Prospective evidence for a hip etiology in patellofemoral pain. Med Sci Sports Exerc 2013;45:1120-1124.

20. Liao TC, Yang N, Ho KY, Farrokhi S, Powers CM. Femur rotation increases patella cartilage stress in females with patellofemoral pain. Med Sci Sports Exerc 2015;47: 1775-1780.

21. Nakagawa TH, Moriya ET, Maciel CD, Serrao FV. Trunk, pelvis, hip, and knee kinematics, hip strength, and gluteal muscle activation during a single-leg squat in males and females with and without patellofemoral pain syndrome. J Orthop Sports Phys Ther 2012;42:491-501.

22. Holden S, Boreham C, Doherty C, Delahunt E. Twodimensional knee valgus displacement as a predictor of patellofemoral pain in adolescent females. Scand J Med Sci Sports 2017;27:188-194.

23. Boling MC, Nguyen AD, Padua DA, Cameron KL, Beutler A, Marshall SW. Gender-specific risk factor profiles for patellofemoral pain. Clin J Sport Med 2021;31: 49-56.

24. Boling M, Padua D. Relationship between hip strength and trunk, hip, and knee kinematics during a jumplanding task in individuals with patellofemoral pain. Int J Sports Phys Ther 2013;8:661-669.

25. Collins NJ, Barton CJ, van Middelkoop M, et al. 2018 Consensus statement on exercise therapy and physical interventions (orthoses, taping and manual therapy) to treat patellofemoral pain: recommendations from the 5 th International Patellofemoral Pain Research Retreat, Gold
Coast, Australia, 2017. Br J Sports Med 2018;52: 1170-1178.

26. Dolak KL, Silkman C, Medina McKeon J, Hosey RG, Lattermann C, Uhl TL. Hip strengthening prior to functional exercises reduces pain sooner than quadriceps strengthening in females with patellofemoral pain syndrome: A randomized clinical trial. J Orthop Sports Phys Ther 2011;41:560-570.

27. Lack S, Barton C, Sohan O, Crossley K, Morrissey D. Proximal muscle rehabilitation is effective for patellofemoral pain: A systematic review with meta-analysis. $\mathrm{Br} J$ Sports Med 2015;49:1365-1376.

28. Piva SR, Goodnite EA, Childs JD. Strength around the hip and flexibility of soft tissues in individuals with and without patellofemoral pain syndrome. J Orthop Sports Phys Ther 2005;35:793-801.

29. Barton CJ, Lack S, Hemmings S, Tufail S, Morrissey D. The 'best practice guide to conservative management of patellofemoral pain': Incorporating level 1 evidence with expert clinical reasoning. Br J Sports Med 2015;49: 923-934.

30. Willy RW, Hoglund LT, Barton CJ, et al. Patellofemoral pain. J Orthop Sports Phys Ther 2019;49:CPGl-CPG95.

31. Argent R, Daly A, Caulfield B. Patient involvement with home-based exercise programs: Can connected health interventions influence adherence? JMIR Mhealth Uhealth 2018;6:e47.

32. Powers CM, Ho KY, Chen YJ, Souza RB, Farrokhi S. Patellofemoral joint stress during weight-bearing and non-weight-bearing quadriceps exercises. J Orthop Sports Phys Ther 2014;44:320-327.

33. Kernozek TW, Gheidi N, Zellmer M, Hove J, Heinert BL, Torry MR. Effects of anterior knee displacement during squatting on patellofemoral joint stress. J Sport Rehabil 2018;27:237-243.

34. Escamilla RF, Zheng N, Macleod TD, et al. Patellofemoral joint force and stress during the wall squat and one-leg squat. Med Sci Sports Exerc 2009;41:879-888.

35. Atkins LT, Smithson C, Grimes D, Heuer N. The influence of sagittal trunk posture on the magnitude and rate of patellofemoral joint stress during stair ascent in asymptomatic females. Gait Posture 2019;74:121-127.

36. Escamilla RF, Zheng N, Macleod TD, et al. Patellofemoral joint force and stress between a short- and long-step forward lunge. J Orthop Sports Phys Ther 2008;38:681-690.

37. Benjaminse A, Welling W, Otten B, Gokeler A. Novel methods of instruction in ACL injury prevention programs, a systematic review. Phys Ther Sport 2015;16:176-186.

38. Salsich GB, Perman WH. Patellofemoral joint contact area is influenced by tibiofemoral rotation alignment in individuals who have patellofemoral pain. J Orthop Sports Phys Ther 2007;37:521-528.

39. Powers CM, Ward SR, Fredericson M, Guillet $M$, Shellock FG. Patellofemoral kinematics during weightbearing and non-weight-bearing knee extension in persons with lateral subluxation of the patella: A preliminary study. J Orthop Sports Phys Ther 2003;33:677-685.

40. Souza RB, Draper CE, Fredericson M, Powers CM. Femur rotation and patellofemoral joint kinematics: A weightbearing magnetic resonance imaging analysis. $J$ Orthop Sports Phys Ther 2010;40:277-285. 
41. Huberti HH, Hayes WC. Patellofemoral contact pressures. The influence of q-angle and tendofemoral contact. J Bone Joint Surg Am 1984;66:715-724.

42. Powers CM. The influence of abnormal hip mechanics on knee injury: A biomechanical perspective. J Orthop Sports Phys Ther 2010;40:42-51.

43. Dinyer TK, Byrd MT, Garver MJ, et al. Low-load vs. highload resistance training to failure on one repetition maximum strength and body composition in untrained women. J Strength Cond Res 2019;33:1737-1744.

44. Liguori G. ACSM's guidelines for exercise testing and prescription. Philadelphia: Wolters Kluwer, 2021.

45. Collins NJ, Bisset LM, Crossley KM, Vicenzino B. Efficacy of nonsurgical interventions for anterior knee pain: Systematic review and meta-analysis of randomized trials. Sports Med 2012;42:31-49.

46. Callaghan MJ, Selfe J. Patellar taping for patellofemoral pain syndrome in adults. Cochrane Database Syst Rev 2012: Cd006717.

47. Barton C, Balachandar V, Lack S, Morrissey D. Patellar taping for patellofemoral pain: A systematic review and meta-analysis to evaluate clinical outcomes and biomechanical mechanisms. Br J Sports Med 2014;48:417-424.

48. Powers CM, Ward SR, Chan LD, Chen YJ, Terk MR. The effect of bracing on patella alignment and patellofemoral joint contact area. Med Sci Sports Exerc 2004;36:1226-1232.

49. Kooiker L, Van De Port IG, Weir A, Moen MH. Effects of physical therapist-guided quadriceps-strengthening exercises for the treatment of patellofemoral pain syndrome: A systematic review. J Orthop Sports Phys Ther 2014;44: 391-402, bl.

50. Chiu JK, Wong YM, Yung PS, Ng GY. The effects of quadriceps strengthening on pain, function, and patellofemoral joint contact area in persons with patellofemoral pain. Am J Phys Med Rehabil 2012;91:98-106.
51. Giles L, Webster KE, McClelland J, Cook JL. Quadriceps strengthening with and without blood flow restriction in the treatment of patellofemoral pain: A double-blind randomised trial. Br J Sports Med 2017;51:1688-1694.

52. Korakakis V, Whiteley R, Epameinontidis K. Blood flow restriction induces hypoalgesia in recreationally active adult male anterior knee pain patients allowing therapeutic exercise loading. Phys Ther Sport 2018;32:235-243.

53. Jayaseelan DJ, Scalzitti DA, Palmer G, Immerman A, Courtney CA. The effects of joint mobilization on individuals with patellofemoral pain: A systematic review. Clin Rehabil 2018;32:722-733.

54. Loudon JK. Biomechanics and pathomechanics of the patellofemoral joint. Int $J$ Sports Phys Ther 2016;11: 820-830.

55. Kim KM, Croy T, Hertel J, Saliba S. Effects of neuromuscular electrical stimulation after anterior cruciate ligament reconstruction on quadriceps strength, function, and patient-oriented outcomes: A systematic review. J Orthop Sports Phys Ther 2010;40:383-391.

56. dos Reis AC, Correa JC, Bley AS, Rabelo ND, Fukuda TY, Lucareli PR. Kinematic and kinetic analysis of the singleleg triple hop test in women with and without patellofemoral pain. J Orthop Sports Phys Ther 2015;45:799-807.

57. Esculier JF, Maggs K, Maggs E, Dubois B. A contemporary approach to patellofemoral pain in runners. $J$ Athl Train 2020;55:0.

58. Dye SF. The knee as a biologic transmission with an envelope of function: A theory. Clin Orthop Relat Res 1996;(325):10-18.

59. Rathleff MS, Graven-Nielsen T, Hölmich P, et al. Activity modification and load management of adolescents with patellofemoral pain: A prospective intervention study including 151 adolescents. Am J Sports Med 2019;47: 1629-1637. 